\title{
Brasilianisches Staatsangehörigkeitsrecht zwischen ius soli und ius sanguinis
}

\author{
Von Jürgen Samtleben, Hamburg*
}

\section{Einleitung}

In Ländern, deren Staatsangehörigkeitsrecht dem strengen ius-soli-Prinzip folgt, stellt sich die Frage, ob und inwieweit für im Ausland geborene Kinder der eigenen Staatsangehörigen der ius-sanguinis-Grundsatz zur Anwendung kommen kann. Die meisten der südamerikanischen Rechte, die traditionell dem ius-soli-Prinzip folgen, stellen für den Erwerb der Staatsangehörigkeit iure sanguinis bei Auslandsgeburten besondere Voraussetzungen auf. Während einige dieser Rechtsordnungen die Registrierung beim zuständigen Konsulat und damit praktisch eine gewisse Heimatverbundenheit des betreffenden Elternteils genügen lassen, verlangt die Mehrzahl eine selbständige Beziehung des Kindes zum „heimischen“ Territorium und macht daher den Erwerb der Staatsangehörigkeit vom späteren Aufenthalt des Kindes in diesem Staat abhängig ${ }^{1}$. In Brasilien schwankt die Rechtsentwicklung zwischen diesen beiden Polen. Die früheren brasilianischen Verfassungen hatten den Erwerb der Staatsangehörigkeit in diesen Fällen zwingend daran geknüpft, dass durch Aufenthalt des Kindes in Brasilien eine Verbindung mit dem nationalen Territorium dokumentiert wurde; auch das Staatsangehörigkeitsgesetz von 1949 beruhte auf dieser Grundlage ${ }^{2}$. Die neuere Entwicklung geht dagegen in andere Richtung.

Noch die Verfassung von 1967 folgte dem traditionellen Grundsatz, dass das im Ausland geborene Kind, ob im Konsulat registriert oder nicht, erst mit der Aufenthaltnahme in Brasilien die brasilianische Staatsangehörigkeit erwirbt, wobei diese nach Volljährigkeit durch eine Option bestätigt werden musste. Bei der Überarbeitung dieser Verfassung im Jahre 1969 wurde die entsprechende Vorschrift nahezu wörtlich übernommen, durch die Versetzung eines Kommas aber in ihr Gegenteil verkehrt. Danach sollte jetzt bereits die Registrierung der Auslandgeburt in der brasilianischen Auslandsvertretung zum endgültigen Erwerb der brasilianischen Staatsangehörigkeit führen. Obwohl diese Auslegung der neuen Verfassungsvorschrift in der Lehre heftige Kritik fand, wurde sie durch die Recht-

* Jürgen Samtleben, Dr. iur., Rechtsanwalt in Hamburg, ehem. Lateinamerika-Referent des Hamburger Max-Planck-Instituts für ausländisches und internationales Privatrecht (1971-2002). Vgl. dazu schon die ältere Übersicht bei Moosmayer, Der Gebietsgrundsatz im Staatsangehörigkeitsrecht (ius soli) unter besonderer Berücksichtigung der südamerikanischen Staaten (1953) 79 ff.

2

Siehe zu den Voraussetzungen nach den Verfassungen von 1824 bis 1946 sowie zum Staatsangehörigkeitsgesetz von 1949 die eingehende Darstellung bei Schmidt/Schlegel, Das Staatsangehörigkeitsrecht von Brasilien und Chile (1957) 11 ff., 90 ff. 
sprechung ausdrücklich bestätigt ${ }^{3}$. Damit war die Entwicklung aber noch nicht zum Abschluss gekommen; der Streit um die richtige Lösung setzte sich vielmehr auch unter der Verfassung von 1988 fort. Während der traditionelle ius-soli-Grundsatz wesentlich mit dem Charakter Brasiliens als Einwanderungsland verknüpft war, zwang die starke Emigration aus Brasilien nunmehr dazu, seine Konsequenzen bei Auslandsgeburten neu zu überdenken.

\section{Verfassung von 1988}

Die brasilianische Verfassung von 1988 übernahm in ihrem Art. 12 die bisherige Regelung mit geringfügigen Änderungen. Ihre Formulierung bestätigte eindeutig die Auslegung, welche die Verfassung von 1969 in der Praxis erfahren hatte. Die entsprechende Vorschrift lautete:

„São brasileiros:

I. natos:

a) $\ldots$.

b) $\ldots$.

c) os nascidos no estrangeiro, de pai brasileiro ou mãe brasileira, desde que sejam registrados em repartição brasileira competente, ou venham a residir na República Federativa do Brasil antes de antingir a maioridade e, alcançada esta, optem em qualquer tempo pela nacionalidade brasileira;“ (Hervorhebung vom Verf.)

Deutsche Übersetzung:

Brasilianer sind:

I. durch Geburt:

a) $\ldots$

b) $\ldots$

c) die im Ausland Geborenen, deren Vater Brasilianer oder deren Mutter Brasilianerin ist, vorausgesetzt daß sie bei der zuständigen brasilianischen Dienststelle im Ausland registriert worden sind, oder daß sie vor Erreichung der Volljährigkeit ihren Aufenthalt im nationalen Territorium nehmen und nach deren Erreichung zu irgendeinem Zeitpunkt für die brasilianische Staatsangehörigkeit optieren.

Die Vorschrift regelte nunmehr klar zwei unterschiedliche Fälle: Die Kinder, die in der brasilianischen Auslandsvertretung registriert werden, erwerben schon auf diese Weise die brasilianische Staatsangehörigkeit. Nur wenn die Registrierung versäumt wurde, bedarf es zum Erwerb der brasilianischen Staatsangehörigkeit der Aufenthaltnahme im brasilianischen Territorium vor Erreichen der Volljährigkeit und der anschließenden Option, wobei 1975, 273-274.

4 Zitiert nach: Senado Federal, Constituição, República Federativa do Brasil (Brasília 1988), S. 16. 
diese anders als nach früherem Recht nicht mehr fristgebunden ist ${ }^{5}$. In der Lehre wurde diese Regelung heftig angegriffen, weil die bloße Registrierung im Ausland keine genügende Bindung mit dem brasilianischen Staat begründen könne; auch die unbefristete Optionsmöglichkeit wurde als unglücklich kritisiert. ${ }^{6}$. Die entsprechende Vorschrift des Staatsangehörigkeitsgesetzes von 1949 wurde weder der Verfassung von 1988 noch den späteren Verfassungsänderungen angepasst, hat aber daneben keine selbständige Bedeutung mehr.

\section{Verfassungsrevision Nr. 3 von 1994}

Diese Kritik kam wenige Jahre später zum Durchbruch in der Verfassungsrevision Nr. 3 vom 7.6.1994, durch welche der Erwerb der brasilianischen Staatsangehörigkeit durch Registrierung im Ausland völlig gestrichen wurde ${ }^{7}$. Nunmehr lautete Art. 12 Verf.: ${ }^{8}$

„São brasileiros:

I. natos:

a) $\ldots$.

b) $\ldots .$.

c) os nascidos no estrangeiro, de pai brasileiro ou de mãe brasileira, desde que venham a residir na República Federativa do Brasil e optem, em qualquer tempo, pela nacionalidade brasileira." (Hervorhebung vom Verf.)

Deutsche Übersetzung: ${ }^{9}$

Brasilianer sind:

I. durch Geburt:

a) $\ldots$

b) $\ldots$

c) die im Ausland Geborenen, die von einem brasilianischen Vater oder einer brasilia-

Dies galt nach der Rechtsprechung auch rückwirkend für laufende Verfahren: Sup.Trib.Fed. 10.11.1988 (RE 103.419), Diário da Justiça v. 25.9.1992. Bereits zuvor wurde die Frist flexibel gehandhabt; vgl. Sup.Trib.Fed. 16.6.1981, Rev.Trim.Jur. 102, 739; Sup.Trib.Just. 2.10.1991, Lex 33, 502 (Altfall).

Dolinger, Direito internacional privado, Parte Geral (2. Aufl. 1993) 145 ff., der es insbesondere für unlogisch hält, dass die Registrierung im Ausland stärker wirken soll als die (der Option vorausgehende) Registrierung im Inland.

Dies beruhte offenbar auf einem Fehler im Abstimmungsverfahren; siehe zu den Beratungen

Trejos Vargas, in: Tiburcio/Barroso (eds), O Direito internacional contemporâneo (2006) 289, $297 \mathrm{ff}$.

8 Zitiert nach "Diário Oficial” vom 9. Juni 1994; ebenso die amtliche Sammlung „Coleção das Leis“ 1994 - VII - 2414.

9

Nach Bergmann/Ferid/Henrich(-Weishaupt), Internationales Ehe- und Kindschaftsrecht, 168.

Lieferung, Stichwort „Brasilien“ (Stand: Oktober 2006) S. 7. 
nischen Mutter abstammen, wenn sie ihren Aufenthalt im nationalen Territorium nehmen und zu irgendeinem Zeitpunkt für die brasilianische Staatsangehörigkeit optieren.

Danach war zum Erwerb der brasilianischen Staatsangehörigkeit nunmehr zwingend die Aufenthaltnahme in Brasilien und die Option für die brasilianische Staatsangehörigkeit erforderlich ${ }^{10}$. Nach der Rechtsprechung ist aber eine solche Option erst nach Begründung eines brasilianischen Aufenthalts und nach Erreichen der Volljährigkeit möglich ${ }^{11}$. Die Übertragung der ausländischen Geburtsurkunde in das brasilianische Zivilregister des Wohnsitzes kann dagegen auch schon für minderjährige Kinder beantragt werden, um dadurch die Aufenthaltnahme nachzuweisen ${ }^{12}$. Dabei wurde nun die Neuregelung ebenso wie zuvor die entsprechende Alternative in der Verfassung von 1988 dahingehend verstanden, dass bereits mit der Aufenthaltnahme in Brasilien die brasilianische Staatsangehörigkeit kraft Geburt erworben wird, diese aber nach Erreichen der Volljährigkeit davon abhängt, dass sie durch die Option bestätigt wird ${ }^{13}$.

Damit war die Rechtsentwicklung wieder zu ihrem Ausgangspunkt in der Verfassung von 1946 zurückgekehrt. Die tatsächlichen Verhältnisse hatten sich aber inwischen gewandelt: War Brasilien früher ein reines Immigrationsland gewesen, so waren seit den achtziger Jahren des 20. Jahrhunderts große Teile der brasilianischen Bevölkerung aus wirtschaftlichen Gründen ins Ausland emigriert, ohne die Verbindung zu ihrem Heimatland aufzugeben $^{14}$. In der konsularischen Praxis vermochte sich die neue Regelung daher nicht durchzusetzen, weil viele der im Ausland lebenden Brasilianer für ihre Kinder brasilianische Passdokumente benötigten. Ein mit dem Justizministerium abgestimmter Erlass des Außenministeriums gestattete deshalb den Konsulaten, die im Ausland geborenen Kinder eines brasilianischen Elternteils weiterhin als „filhos de brasileiro“ in den Pass der Eltern

Dolinger, Direito internacional privado, Parte Geral (6. Aufl. 2001) 166 ff., der eine Befristung vermisst. Den Wegfall der Registrierung im Ausland bedauert Sup.Trib.Just. 20.6.1995, Lex 77, 372 (374 f.).

11 Sup.Trib.Fed. 22.3.2005, Rev.Trim.Jur. 194, 1069; Trib.Reg.Fed.2a.Reg. 3.12.2003, Rev.Trib. 825, 409; Trib.Reg.Fed.4a.Reg. 2. und 16.3.2005, Lex 191, 527 und 194, 535; siehe auch Sup.Trib.Fed. 31.8.2000, Rev.Trim.Jur. 177, 562 (564): gemeinsame Option von Kind und volljährigem Enkel.

Grundlage dafür ist das Gesetz Nr. 6.015 von 1973 über die öffentlichen Register, Art. $32 \S 2$. Siehe dazu aus der Rechtsprechung schon Sup.Trib.Just. 1.6.1993, Rev.Trib. 705, 209; 10.9.1997, Lex 103, 36 (40), wo dies als ,provisorische Option“ bezeichnet wird; ebenso Trib.Fed.Reg.5a.Reg. 3.2.2004, Rev.Trib. 827, 483; weitere Nachweise bei Trejos Vargas (oben Fn. 7), 309 f. Zuständig sind nach dieser Rechtsprechung die Bundesgerichte; anders jetzt Trib.Fed.Reg.1a.Reg. 24.4.2006, Rev.Trib. 852, 375 und Sup.Trib.Just. 9.8.2006 (CC 58743), Diário da Justiça v. 21.8.2006.

Sup.Trib.Fed. 25.9.2003, Rev.Trim.Jur. 188, 753; 22.3 .2005 (Fn. 11); 23.8.2005, Rev.Trib. 843, 180: Option als „aufschiebende Bedingung“; näher dazu Ferreira Gouvêa, Rev.Trib. 685 (1992) $252 \mathrm{ff}$. 
einzutragen oder ihnen einen eigenen Pass auszustellen, jedoch nur bis zum Erreichen der Volljährigkeit $^{15}$. Auch enthielten die Pässe in diesen Fällen den ausdrücklichen Vermerk, dass die brasilianische Staatsangehörigkeit der Bestätigung durch Aufenthaltnahme in Brasilien und Option bedürfe ${ }^{16}$. Die dadurch erzeugte Rechtsunsicherheit führte dazu, dass diese Kinder vielfach entgegen der Verfassungsvorschrift als brasilianische Staatsangehörige kraft Geburt angesehen wurden. Die in der Verfassung geforderte Aufenthaltnahme in Brasilien und Option wurde dabei nicht als Erwerbstatbestand der Staatsangehörigkeit, sondern als eine für deren Beibehaltung notwendige Rechtshandlung verstanden ${ }^{17}$.

\section{Verfassungsänderung Nr. 54 von 2007}

Diese Diskrepanz zwischen dem geschriebenen Recht und der Rechtspraxis führte zu verschiedenen parlamentarischen Initiativen zur Reform des Staatsangehörigkeitsrechts ${ }^{18}$. Durch die Verfassungsänderung Nr. 54 vom 20.9.2007 wurde schließlich Art. 12 der Verfassung wieder an die Formulierung von 1988 angeglichen und lautet nunmehr: ${ }^{19}$

„São brasileiros:

I. natos:

a) $\ldots$.

b) $\ldots$.

c) os nascidos no estrangeiro de pai brasileiro ou mãe brasileira, desde que sejam registrados em repartição brasileira competente ou venham a residir na República Federativa do Brasil e optem, em qualquer tempo, depois de atingida a maioridade, pela nacionalidade brasileira." (Hervorhebung vom Verf.)

Deutsche Übersetzung: ${ }^{20}$

Brasilianer sind:

I. durch Geburt:

a) $\ldots$

b) $\ldots$

c) die im Ausland Geborenen, die von einem brasilianischen Vater oder einer brasilia-

Dolinger (oben Fn. 10), 168 ff.; eingehend dazu Moll, in: Tiburcio/Barroso (eds.), O Direito internacional contemporâneo (2006) 627, 634 f.; vgl. auch Trejos Vargas (oben Fn. 7), 289, 307 f.

"A condiçao de brasileiro está sujeita à confirmação através de dois eventos: residência no Brasil e opção pela nacionalidade brasileira perante Juiz Federal." (Ein entsprechender Eintrag liegt dem Verf. vor.)

17

Vgl. Bergmann/Ferid/Henrich (-Weishaupt) (oben Fn. 9), S. 6 f.

18

Zu den einzelnen Gesetzesvorlagen und den Beratungen siehe Moll (oben Fn. 15), S. 635 ff.

19

Zitiert nach "Diário Oficial” vom 21. September 2007.

20

Nach Bergmann/Ferid/Henrich(-Weishaupt), Internationales Ehe- und Kindschaftsrecht, 177. Lieferung, Stichwort „Brasilien“ (Stand: 30.4.2008) S. 8. 
nischen Mutter abstammen, soweit sie in der zuständigen brasilianischen Auslandsvertretung registriert sind oder ihren Aufenthalt in der Föderativen Republik Brasilien nehmen und zu irgendeinem Zeitpunkt nach Erreichung der Volljährigkeit für die brasilianische Staatsangehörigkeit optieren. (Hervorhebung vom Verf.)

Bei näherer Betrachtung fällt jedoch auf, dass wiederum die Interpunktion gegenüber der ursprünglichen Fassung verändert worden ist. Während nach der Verfassung von 1988 die beiden Fälle des Staatsangehörigkeitserwerbs durch ein Komma getrennt und damit nur bei fehlender Registrierung im Ausland kumulativ die Aufenthaltnahme in Brasilien und die Option für die brasilianische Staatsangehörigkeit verlangt wurden, ist dieses Komma jetzt entfallen; die einzelnen Voraussetzungen des Staatsangehörigkeitserwerbs sind lediglich durch ein ,oder“ sowie ein ,und“ miteinander verbunden. Nach der bisherigen Geschichte dieser Verfassungsvorschrift ist anzunehmen, dass der Wegfall des Kommas nicht ohne Bedacht erfolgt ist. Die jetzige Gleichordnung der einzelnen Voraussetzungen deutet darauf hin, dass die Registrierung im Ausland und die Aufenthaltnahme in Brasilien zwei selbständige Fälle des Staatsangehörigkeitserwerbs bilden, dass aber in beiden Fällen nach Erreichen der Volljährigkeit eine Option für die brasilianische Staatsangehörigkeit erforderlich ist ${ }^{21}$. Diese Gleichordnung kommt auch in einer neu eingefügten Übergangsvorschrift zum Ausdruck: Die zwischen dem 7.6.1994 und der Verkündung der jetzigen Verfassungsänderung im Ausland geborenen Kinder eines brasilianischen Elternteils können in der zuständigen Auslandsvertretung oder bei späterem Aufenthalt in Brasilien dort registriert werden (Art. 95 der Disposições Constitucionais Transitórias). Damit wird rückwirkend der Registrierung im Ausland die gleiche - aber keine stärkere - Wirkung beigelegt wie der Registrierung am brasilianischen Wohnsitz.

Überträgt man die Grundsätze der oben genannten Rechtsprechung ${ }^{22}$ auf die jetzige Fassung, so ergibt sich daher das folgende Bild: Das im Ausland geborene Kind eines brasilianischen Elternteils erlangt die brasilianische Staatsangehörigkeit kraft Geburt, wenn es entweder in der brasilianischen Auslandsvertretung registriert wird oder seinen Aufenthalt in Brasilien nimmt. Als Nachweis der Staatsangehörigkeit dient im ersten Fall die Eintragung in den Pass, im letzteren Fall der vom Gericht des brasilianischen Wohnsitzes aufgrund der ausländischen Geburtsurkunde vorgenommene Geburtseintrag ${ }^{23}$. Nach Erreichen der Volljährigkeit (die nach dem neuen Zivilgesetzbuch von 2002 mit 18 Jahren erworben wird) wird der Betreffende aber nur als brasilianischer Staatsangehöriger kraft

Anders Weishaupt (oben Fn. 12), S. 7, der die Option nur bei fehlender Registrierung im Konsulat für notwendig hält; siehe dagegen schon das Argument bei Dolinger (oben Fn. 6).

22

Oben Fn. 13.

23

Siehe dazu oben Fn. 12. Die Registrierung im Konsulat berechtigt aber nicht zur Registrierung in Brasilien, wenn dort kein Aufenthalt nachgewiesen ist; Trib.Reg.Fed.4a.Reg. 12.12.2007, Rev.Trib. 871, 401. 
Geburt angesehen, wenn er die entsprechende Option ausgeübt hat ${ }^{24}$. Diese muss nach Art. $109, \mathrm{X}$ der Verfassung vor einem Bundesgericht erklärt und von diesem bestätigt werden ${ }^{25}$.

\section{4. $\quad$ Fazit}

Im Spannungsfeld zwischen dem ius soli und dem ius sanguinis hat das brasilianische Recht damit nach langen Auseinandersetzungen einen sinnvollen Ausgleich gefunden. Dem alten Grundsatz des ius soli, der Brasilien als Einwanderungsland charakterisiert, entspricht es, wenn bei im Ausland geborenen Kindern brasilianischer Eltern zum Erwerb der Staatsangehörigkeit die Aufenthaltnahme in Brasilien verlangt wird, um einen Bezug mit dem nationalen Territorium herzustellen. Nachdem Brasilien in den letzten Jahrzehnten auch zum Auswanderungsland geworden ist, erfordert der Grundsatz des ius sanguinis, der die fortbestehende Bindung zum Heimatstaat verkörpert, dass den im Ausland lebenden Brasilianern eine Möglichkeit eröffnet wird, ihren Kinder durch Registrierung im Konsulat die brasilianische Staatsangehörigkeit zu sichern. In beiden Fällen muss aber das volljährige Kind sich durch Option zu Brasilien bekennen, um die Staatsangehörigkeit beizubehalten. 18.2.2008: Eine in Brasilien registrierte Auslandsgeburt begründet keine brasilianische Staatsangehörigkeit, wenn keine Option des inzwischen Volljährigen nachgewiesen ist.

Sup.Trib.Fed. 25.9.2003 (oben Fn. 13); Sup.Trib.Just. 10.9.1997 (oben Fn. 12). 